\title{
ABSOLUTE ZERO DIVISORS AND LOCAL NILPOTENCE IN ALTERNATIVE ALGEBRAS
}

\author{
KEVIN MCCRIMMON ${ }^{1}$
}

ABSTRACT. It has been conjectured that absolute zero divisors generate locally nilpotent ideal s in Jordan and altern ative al gebras. A. M. Slin'ko has recently established this result for special Jordan algebras; in this note we show how his method can be modified to establish the result for alternative algebras.

Throughout we consider alternative algebras $\mathfrak{U}$ over an arbitrary ring of scalars $\Phi$. Recall that an element $z$ of $\mathfrak{U}$ is an absolute zero divisor if $z$ U $z=0$. An algebra is locally nilpotent if every finitely-generated subalgebra is nilpotent. The strong semiprime radical $S(\mathfrak{U})$ is the smallest ideal $\mathscr{B}$ of $\mathfrak{U}$ such that $\mathscr{U} / \mathscr{B}$ is strongly semiprime (contains no absolute zero divisors), and the Levitzki or locally nilpotent radical $L(\mathfrak{U})$ is the smallest ideal $\mathscr{B}$ such that $\mathscr{U} / \mathbb{B}$ contains no locally nilpotent ideals.

Slinko's Jordan result is used to establish

Lemma. If an alternative algebra $\mathfrak{U}$ is generated by absolute zero divisors, then the algebra $\mathfrak{L}(\mathfrak{U})$ of left multiplications of $\mathfrak{Q}$ is locally nilpotent.

Proof. The space $J=\left\{L_{x} \mid x \in \mathfrak{U}\right\}$ of left multiplications is a special Jordan subalgebra of End(U), since by the left Moufang formula $L_{x} L_{y} L_{x}=L_{x y x}$ and $L_{x} L_{x}=L_{x} 2$. The algebra $\mathcal{L}(\mathfrak{U})$ is the associative envelope of $J$ in End( (U)).

If $z$ is an absolute zero divisor in $\mathcal{U}$, then $L_{z}$ is an absolute zero divisor in $J: L_{z} L_{x} L_{z}=L_{z x z}=0$. Any monomial having an absolute zero divisor as a factor is itself an absolute zero divisor (using the fundamental formulas $U_{x y}=L_{x} U_{y} R_{x}=R_{y} U_{x} L_{y}$ repeatedly to break up the $U$-operator of the monomial until a $U_{z}=0$ is reached), so if the absolute zero divisors generate $\mathcal{U}$ they actually span $\mathcal{U}$. Now it is not in general true that if $z_{i}$

Received by the editors December 18, 1973.

AMS (MOS) subject classifications (1970). Primary 17D05; Secondary 17C50.

Key words and phrases. Absolute zero divisor, locally nilpotent, alternative algebra.

1 The author wishes to thank the NSF for its support of this and previous work. 
generate $\mathcal{O}$ then the $L_{z_{i}}$ generate $J$ and $\mathcal{L}(\mathfrak{U})$, but it is clear that if the $z_{i}$ span Of then the $L_{z_{i}}$ span $J$ and generate $\mathscr{L}(\mathscr{U})$. Thus $J$ is spanned by absolute zero divisors. By Slinko's Jordan theorem [2, pp. 713-714] the associative envelope $\mathfrak{L}(\mathfrak{U})$ of $J$ is locally nilpotent.

We are now ready to state the alternative version of Slinko's theorem.

Slinko's theorem I. An alternative algebra which is generated by absolute zero divisors is locally nilpotent.

Proof. Let $\mathfrak{Z}$ be a finitely-generated subalgebra of an algebra $\mathfrak{U}$ generated by absolute zero divisors $z_{i}$; we must prove $B$ is nilpotent. Now each of the finitely many generators of $B$ is by hypothesis a polynomial in a finite number of the absolute zero divisors $z_{i}$, so $B$ is contained in the subalgebra (5) generated by all the se finitely many $z_{i}$. It suffices to prove (5) is nilpotent. Thus the theorem is equivalent to

Slinko's theorem II. An alternative algebra generated by a finite number of absolute zero divisors is nilpotent.

Proof. Suppose $\mathfrak{A}$ is generated by absolute zero divisors $z_{1}, \cdots, z_{n}$. We know [1, Proposition 3, p. 290] that every element of $\mathfrak{U}^{k}$ is a linear combination of "2nd order monomials" of the form $w_{1}\left(w_{2}\left(\cdots w_{r}\right)\right.$ ), where each $w_{i}=z_{i_{1}}\left(z_{i_{2}}\left(\cdots z_{i_{s}}\right)\right)$ is a "1st order monomial" in the generators $z_{j}$, and the degrees of the $w_{i}$ add up to at least $k: \partial w_{1}+\partial w_{2}+\cdots+\partial w_{r} \geq k$. By the lemma there is an $N=N\left(z_{1}, \cdots, z_{n}\right)$ such that

$$
L_{z_{1}} L_{z_{i_{2}}} \cdots L_{z_{i_{s-1}}}=0 \text { for } s \geq N \text {, }
$$

so

$$
w_{i}=L_{z_{i_{1}}} L_{z_{i_{2}}} \cdots L_{z_{i_{s-1}}}\left(z_{i_{s}}\right)=0
$$

if $\partial w_{i}=s \geq N$. Consider the finite number $w_{1}, \cdots, w_{m}$ of 1st order monomials $w_{i}$ of degree $<N$. Once more by the lemma there is an $M=$ $M\left(w_{1}, \cdots, w_{m}\right)$ such that

$$
w_{i_{1}}\left(w_{i_{2}}\left(\cdots w_{i_{r}}\right)\right)=L_{w_{i_{1}}} L_{w_{i_{2}}} \cdots L_{w_{i_{r-1}}}\left(w_{i_{r}}\right)=0 \text { for } r \geq M \text {. }
$$

But then $\mathfrak{Q}^{N M}$ is spanned by $w_{1}\left(w_{2}\left(\cdots w_{r}\right)\right)$ for $\partial w_{1}+\cdots+\partial w_{r} \geq N M$, where this monomial vanishes unless all $\partial w_{i}<N\left(w_{i}=0\right.$ if $\left.\partial w_{i} \geq N\right)$, and if all $\partial w_{i}<N$ then $r N>\partial w_{1}+\cdots+\partial w_{r} \geq N M$ implies $r \geq M$, so $w_{1}\left(w_{2}\left(\cdots w_{r}\right)\right)=0$ anyway. Thus $\mathfrak{U}^{N M}=0$ and $\mathfrak{O}$ is nilpotent. $\square \square$ 
From this we can immediately derive some corollaries.

Corollary. The ideal Z(U) generated (or spanned) by the absolute zero divisors is contained in the Levitzki radical, $Z(\mathfrak{U}) \subset L(\mathfrak{U})$.

Corollary. If $\mathrm{U}$ contains no locally nilpotent ideals, it is strongly semiprime.

Corollary. $S(\mathscr{U}) \subset L(\mathscr{U})$.

Thus in an arbitrary alternative algebra we have the chain of radicals

$$
P(\mathfrak{U}) \subset S(\mathfrak{U}) \subset L(\mathfrak{U}) \subset N(\mathfrak{U}) \subset J(\mathfrak{U})
$$

for $P$ the prime radical, $N$ the nil radical, and $J$ the Jacobson-Smiley radical.

Since simple algebras are not locally nilpotent they have no locally nilpotent ideals, hence

Corollary. A simple alternative algebra contains no absolute zero divisors.

Of course, this also follows from inspecting the known classification of simple alternative algebras.

Another result which follows without appeal to the classification of simple algebras is the

Corollary. A simple, commutative, alternative algebra is a field.

Proof. It suffices to prove associativity. A simple commutative alternative algebra cannot contain nilpotent elements, for if it contained nilpotent elements it would contain elements with $z^{2}=0$, and by commutativity such $z$ would be absolute zero divisors $\left(z x z=z z x=z^{2} x=0\right.$ for all $x$ ), contrary to our previous corollary. But any associator is nilpotent, $[x, y, z]^{2}$ $=0$, so all associators must vanish and the algebra is associative.

Yet another result whose proof can be simplified is one due to Zhevlakov, $\mathrm{Ng}$, and others:

Corollary. If $\mathrm{U}$ is a simple alternative algebra, then $\mathrm{Q⿱}^{+}$is simple as a quadratic Jordan algebra.

Proof. Suppose $B$ were a proper Jordan ideal of $\mathfrak{U}^{+}$. Then its kernel (the largest alternative ideal contained in $B$ ) must be zero by simplicity of थ. Now it is easy to verify that in general 
$\operatorname{Ker} \mathscr{B}=\{b \in \mathscr{B} \mid b \mathfrak{Q} \subset \mathscr{P}\}=\{b \in \mathscr{B} \mid \mathscr{U} b \subset \mathbb{B}\}$

for a Jordan ideal $\mathbb{B}$, hence

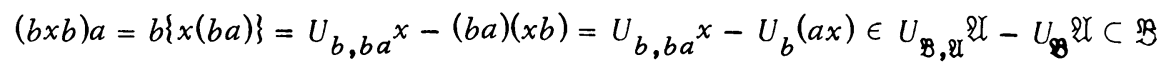

shows $U_{\mathfrak{B}} \mathfrak{A} \subset \operatorname{Ker} \mathfrak{B}$. Thus when the kernel is zero, all elements of $\mathbb{B}$ are absolute zero divisors, and therefore by the corollary, $\mathbb{B}=0$.

Remark. The result we really want, of course, is $P(\mathfrak{U})=S(\mathfrak{U})$ rather than just $S(\mathscr{U}) \subset L(\mathscr{U})$; we want to know that a semiprime alternative algebra contains no absolute zero divisors so that strong semiprimeness is equivalent to semiprimeness. Kleinfeld [3] has proved this for characteristic $\neq 3$ situations (where $\mathfrak{U}$ has no 3-torsion or $3 \mathfrak{U}=\mathfrak{U}$ ), but no one has been able to extend it to the general case. This is the main stumbling block to a classification of prime and semiprime alternative algebras.

For many applications (such as the above corollaries) the weaker inclusion $S(\mathscr{U}) \subset L(\mathscr{U})$ suffices. In characteristic $\neq 3$ the corollaries also follow from Kleinfeld's result.

Further remark. Profess or Michael Slater has pointed out that Slinko's theorem can also be derived quickly from the known structure the ory of alternative rings. The advantage of the present direct proof is that it bypasses the difficult part of the structure theory (due to Shirshov) concerning p.i. rings; indeed, it can be used in place of Shirshov's work to derive that structure theory (as will appear in a forthcoming paper of Professor Slater). It seems that the really essential part of the Shirshov machinery is the part used in proving Slinko's theorem (see the lemma below).

Slinko's proof was given for linear Jordan algebras over a field of characteristic $\neq 2$. His proof can be modified to work for quadratic Jordan algebras over an arbitrary ring of scalars. To make this paper self-contained we will repeat (and thereby translate from Russian) the part of Slinko's proof applying to alternative algebras, making the minor modifications necessary for the case of an arbitrary ring of scalars.

Lemma. Let $z_{1}, \cdots, z_{n}$ be absolute zero divisors in an alternative algebra. Then there is an integer $N=2^{n}(n+1)$ ! sucb that any product $L_{z_{i}} \cdots L_{z_{i}}$ of left multiplications $L_{z_{i}}$ of length $N$ vanishes.

Proof. We induct on $n, n=0$ being vacuous. Assume the result for $n-1$ absolute zero divisors, so any monomial in $L_{z_{1}}, \cdots, L_{z_{n-1}}$ of length 
$N_{0}=2^{n-1} n$ ! vanishes. We claim that any monomial in $L_{z_{1}}, \cdots, L_{z_{n}}$ of length $N=2^{n}(n+1)$ ! vanishes.

We can write any such monomial as

$$
w\left(L_{z_{1}}, \cdots, L_{z_{n}}\right)=L_{z_{i_{1}}} \cdots L_{z_{i}}
$$

where $w\left(x_{1}, \cdots, x_{n}\right)=x_{i_{1}} \cdots x_{i_{N}}$ is a word on the alphabet of letters $x_{1}, \cdots, x_{n}$. We order this alphabet in the natural way, $x_{1}<x_{2}<\cdots<x_{n}$, and induct on the lexicographic order of $w$. This induction gets off the ground since the lexicographically lowest word of length $N$ is $x_{1}^{N}$, and $L_{z_{1}}^{N}=L_{z_{1}^{N}}=0$ by $z_{1}^{e}=z_{1}\left(z_{1}^{e-2}\right) z_{1}=0$ if $e \geq 3$ and $z_{1}$ is an absolute zero divisor.

Assume the result for lexicographically lower words $w^{\prime}$. Write

$$
w=w_{0}{ }_{n}^{e}{ }_{n}^{1} w_{1} \cdots w_{r-1}{ }^{e}{ }_{n}{ }^{r} w_{r}
$$

for exponents $e_{i} \geq 1$ and words $w_{i}\left(x_{1}, \cdots, x_{n-1}\right)$ not involving $x_{n}$ where $w_{1}, \cdots, w_{r-1}$ are nonempty (we allow $w_{0}=1$ or $w_{r}=1$ ). The word $w$ will vanish when evaluated at the $L_{z_{i}}, w\left(L_{z_{1}}, \cdots, L_{z_{n}}\right)=0$, if any exponent $e_{i} \geq 3$ (since $L_{z_{n}}^{e}=L_{z_{n}^{e}}=0$ if $e \geq 3$ ) or if any word $w_{i}$ has length $\geq N_{0}$ (by the induction hypothesis for $n-1$ absolute zero divisors). Thus we may assume $\boldsymbol{e}_{i} \leq 2$ and $\partial w_{i}<N_{0}$. Then

$$
\begin{aligned}
(n+1) 2^{n} n ! & =N=\partial w=\sum_{1}^{r} e_{i}+\sum_{0}^{r} \partial w_{i} \leq \sum_{1}^{r} 2+\sum_{0}^{r} N_{0} \\
& =2 r+(r+1) N_{0}<(r+1)\left(2+N_{0}\right) \leq(r+1) 2 N_{0}=(r+1) 2^{n} n !
\end{aligned}
$$

forces $n<r$.

If one of the $w_{i}$ for $1 \leq i \leq r-1$ has degree 1 , say $w_{i}=x_{j}$, then again $w\left(L_{z_{1}}, \cdots, L_{z_{n}}\right)=0$ since already

$$
\begin{aligned}
& L_{z_{n}}^{e_{i}} L_{z_{j}}{ }^{L_{z}}{ }^{e_{i+1}}=L_{z_{n}}^{e_{i}^{-1}}\left(L_{z_{n}} L_{z_{j}}{ }^{L} z_{n}\right) L_{z_{n}}^{e^{i+1}-1} \\
& =L_{z_{n}}^{e}{ }^{-1}\left(L_{z_{n} z_{n} z_{n}}\right) L_{z_{n}}^{e}{ }_{i+1}^{-1}=0
\end{aligned}
$$

if $z_{n}$ is an absolute zero divisor. Thus we may assume the monomials $w_{1}, \cdots, w_{r-1}$ have degree $\geq 2$; there are $r-1 \geq n$ of the se monomials and only $n-1$ variables $x_{1}, \cdots, x_{n-1}$ appearing in them, so two of them must end in the same variable $x_{k}: w_{i}=w_{i}^{\prime} x_{k}, w_{j}=w_{j}^{\prime} x_{k}$ for $1 \leq i<j \leq r-1$. We 
will rearrange the letters betwe en these two $x_{k}$, obtaining lexicographically lower words.

By $\left[1\right.$, p. 286] there is a Jordan monomial $p\left(x_{1}, \cdots, x_{n}\right)$ having as lexicographically leading monomial

$$
v=x_{n}{ }^{e}{ }^{i+1} w_{i+1} \cdots x_{n}{ }^{e}{ } w_{j}^{\prime}
$$

(here it is crucial that $v$ begins with an $x_{n}$ and ends with a lower letter, since $w_{j}=w_{j}^{\prime} x_{k}$ for degree $\geq 2$ implies $w_{j}^{\prime}$ is of degree $\geq 1$ on the letters $\left.x_{1}, \cdots, x_{n-1}\right)$; we write

$$
p\left(x_{1}, \cdots, x_{n}\right)=v+\sum v_{a}
$$

for associative words $v_{\alpha}$ lexicographically lower than $v$. Then $w=w^{\prime} v w^{\prime \prime}$ for

$$
w^{\prime}=w_{0} w_{n}^{e}{ }^{1} \cdots x_{n}^{e}{ }^{i} w_{i}=\left(w_{0} \cdots w_{i}^{\prime}\right) x_{k}=u^{\prime} x_{k}
$$

and

$$
w^{\prime \prime}=x_{k}{ }_{n}^{e}{ }_{j+1} w_{j+1} \cdots x_{n}^{e}{ }_{r} w_{r}=x_{k} u^{\prime \prime}
$$

where

$$
\begin{aligned}
w^{\prime} p\left(L_{z_{1}}, \cdots, L_{z_{n}}\right) w^{\prime \prime} & =u^{\prime} L_{z_{k}} p\left(L_{z_{1}}, \cdots, L_{z_{n}}\right) L_{z_{k}} u^{\prime \prime} \\
& =u^{\prime} L_{z_{k}} L_{p\left(z_{1}, \ldots, z_{n}\right) L_{z_{k}} u^{\prime \prime} \quad(\text { by }[1, \text { Proposition 2, p. 285]) }} \\
& =u^{\prime} L_{z_{k} p\left(z_{1}, \ldots, z_{n}\right) z_{k}} u^{\prime \prime}=0
\end{aligned}
$$

if $z_{k}$ is an absolute zero divisor. This allows us to replace $v$ by the lower $v_{a}$ in $w$,

$$
\begin{aligned}
w\left(L_{z_{1}}, \cdots, L_{z_{n}}\right) & =w^{\prime} v\left(L_{z_{1}}, \cdots, L_{z_{n}}\right) w^{\prime \prime} \\
& =w^{\prime} p\left(L_{z_{1}}, \cdots, L_{z_{n}}\right) w^{\prime \prime}-\sum w^{\prime} v_{\alpha}\left(L_{z_{1}}, \cdots, L_{z_{n}}\right) w^{\prime \prime} \\
& =-\sum w^{\prime} v_{\alpha}\left(L_{z_{1}}, \cdots, L_{z_{n}}\right) w^{\prime \prime} \\
& =-\sum w_{\alpha}\left(L_{z_{1}}, \cdots, L_{z_{n}}\right)
\end{aligned}
$$

for $w_{a}=w^{\prime} v_{a} w^{\prime \prime}$ lexicographically lower than $w=w^{\prime} v w^{\prime \prime}$ (but still of the same length $N)$. By lexicographic induction these $w_{a}\left(L_{z_{1}}, \cdots, L_{z_{n}}\right)=0$, 
hence $w\left(L_{z_{1}}, \ldots, L_{z_{n}}\right)=0$ too. This completes both inductions, so any $L_{z_{i_{1}}} \cdots L_{z_{i_{N}}}=0$.

\section{REFERENCES}

1. K. McCrimmon, Alternative algebras satisfying polynomial identities, J. Algebra 24 ( 1973), 283-292.

2. A. M. Slin'ko, On the Jacobson radical and absolute zero divisors in special Jordan algebras, Algebra i Logika 11 (1972), 711-723. (Russian)

3. E. Kleinfeld, Alternative nil rings, Ann. of Math. (2) 66 (1957), 395-399. MR 19, 383.

DEPARTMENT OF MATHEMATICS, UNIVERSITY OF VIRGINIA, CHARLOTTESVILLE, VIRGINIA 22901 